\title{
Quality control on radiant heaters manufacture
}

\author{
Daniel A. González ${ }^{*}$, Francisco J. Madruga, Clemente Ibarra-Castanedo ${ }^{b}$, Olga Conde, Jose M. \\ López-Higuera \\ Photonic Engineering Group, University of Cantabria, ETSIIT - Avda. Los Castros s/n, 39005 \\ Santander, Spain \\ ${ }^{b}$ Computer Vision and Systems Laboratory, Université Laval, G1K7P4 Québec, Canada
}

\begin{abstract}
An inspection process of radiant heaters is presented in this paper. The proposed non destructive testing and evaluation (NDT\&E) technique for defect assessment of radiant heaters is based on infrared thermography images properly acquired and processed. The technique can be used in on-line fabrication quality control radiant heaters manufacturing processes. By exciting the heater with a very short electrical pulse, a sequence of thermographic images is captured by an infrared camera and then analyzed. Regardless of the electrical excitation applied to the heating element of the heater, the electrical power supplied will dissipate at the resistor. Provided enough spatial resolution, the heaters could be tested with an infrared camera capturing the radiated heat. The analysis of the heating wire during the heating flank shows differences among pixels corresponding to defective points and pixels belonging to non-defective areas of the wire. The automation is provided by the development of an algorithm that looks for the siope of the heating evolution of each pixel. A Radon Transform based algorithm is here proposed to reduce human intervention providing just one image where an operator could quickly locate possible defects.
\end{abstract}

Keywords: Infrared Thermography, Image Processing, Radon Transform, defect detection, radiant heaters, quality control.

\section{INTRODUCTION}

Nowadays, when technology comes out in a lot of each day more sophisticated tools, the demand of quality control has become one of the most important goals in any industry. The researchers' efforts are currently underway to develop new innovative techniques able to help operators to decide whether defects are to be found or not. Non-Destructive Evaluation and Testing tecbniques (NDET) have been developed and improved during years becoming fast, non-contact and safe. These are the advantages of Infrared Thermography (IT) over other NDET techniques such as ultrasounds or $\mathrm{X}$-rays [1].

In the case under study, the niche of application corresponds to manufacturing industries of radiant heaters. At this point is interesting to detect defects on the heaters that could lead to a lack of effectiveness or even malfunction or breakdown. IT represents the easiest NDET to be used to. The supplied electrical power is expected to be uniformly dissipated in the heater resistor. Under any excitation, the heating and cooling behaviours of any two parts of the heating wire should be the same. These behaviours can be observed by the photodetectors or thermal detectors of infrared cameras and, consequently, the heaters could be perfectly tested by using an infrared camera when enough spatial resolution is provided $[2,3]$.

Here, a transient behaviour produced on heaters is analyzed. The time consumed for each inspection is just the time invested on image processing. The excitation on the heater is controlled by an electronic timer and can be shorter than $0.1 \mathrm{sec}$ although it should be long enough and always with enough energy to produce the heating. This excitation is consequently modelled as a quasi instantaneous pulse (ideally it would correspond to a Dirac's Delta function). Considering Joule's law (the amount of heat liberated has a quadratic dependency of the supplied electrical current but linear with time) and recording the heating by a fast infrared camera, each pixel on the image corresponding to a point

dagonfer@ateisa.unican.es; phone/fax: +34 942 200877; www.teisa.unican.es/gif

Thermosense XXVIII, edited by Jonathan J. Miles, G. Raymond Pøacock, Kathryn M. Knettel, Proc. of SPIE Vol. 6205. 620510, (2006) -0277-786X106/\$15 - doi: 10.1117/12.665490 
the heating element acts in a linear way. If any defect exists, its behaviour linearity will be different and therefore detectable.

In this paper, a description of the setup and the used processing technique for a quality contol of radiant heaters is presented. A Radon Transform based algorithm is proposed to reduce human intervention providing just one image where an operator can quickly locate the potential defects. The performance in defect detection has been evaluated using commercial pieces containing a range of real and artificial defects.

\section{INSPECTION PROCESS}

Real commercial specimens, provided by a manufacturer of radiant heaters, with a range of real and artificial defects were heated using an electrical control that offers a fast and precise energy shut off. The inspection is performed on a proposed setup that comprises a specific and custom electronic timer, an infrared camera and the sample, a radiant heater. A scheme of the setup is shown in Figure 1.

The duration of the excitation is controlled by a Crozet timer, in function $\mathrm{H}$, which energizes an output contact during a time that is adjustable. The value of interest for the timer duration was centred on short pulses as closer to the ideal Dirac's Delta function as possible $(0.1 \mathrm{sc})$ but always assuring enough energy to heat the wire. This fact was considered because the electrical power dissipated on heating element is dominated by the radiance mechanism instead of being the equilibrated contribution of the different heat transfer mechanisms (natural convection, conduction and radiation mechanisms). During the very first part of the heating, natural convection and thermal conduction between the wire and the reflective shield (a low thermal-diffusivity substrate) could be neglected since they are linearly proportional to the temperature difference between the wire and the ambient. However, radiance is proportional to the fourth power of the temperature difference.

The inspection includes the heating tendency of the wire and is captured by an infrared camera with specific characteristics. A low thermal sensitivity or noise equivalent power (NEP) of the camera is desirable to measure the expected low increases of wire temperature. Also, due to work with transient timing of only $0.1 \mathrm{sec}$, fast cameras are ineeded to obtain several frames in such a heat transient. In this case, a CMT 128M Thermosensorik GmbH camera has been used due to its high frame rate and excellent temperature resolution (NETD $<20 \mathrm{mK}$ ). Free sizeable subframes could be obtained up to a maximum subframe rate higher than $5 \mathrm{kHz}$.

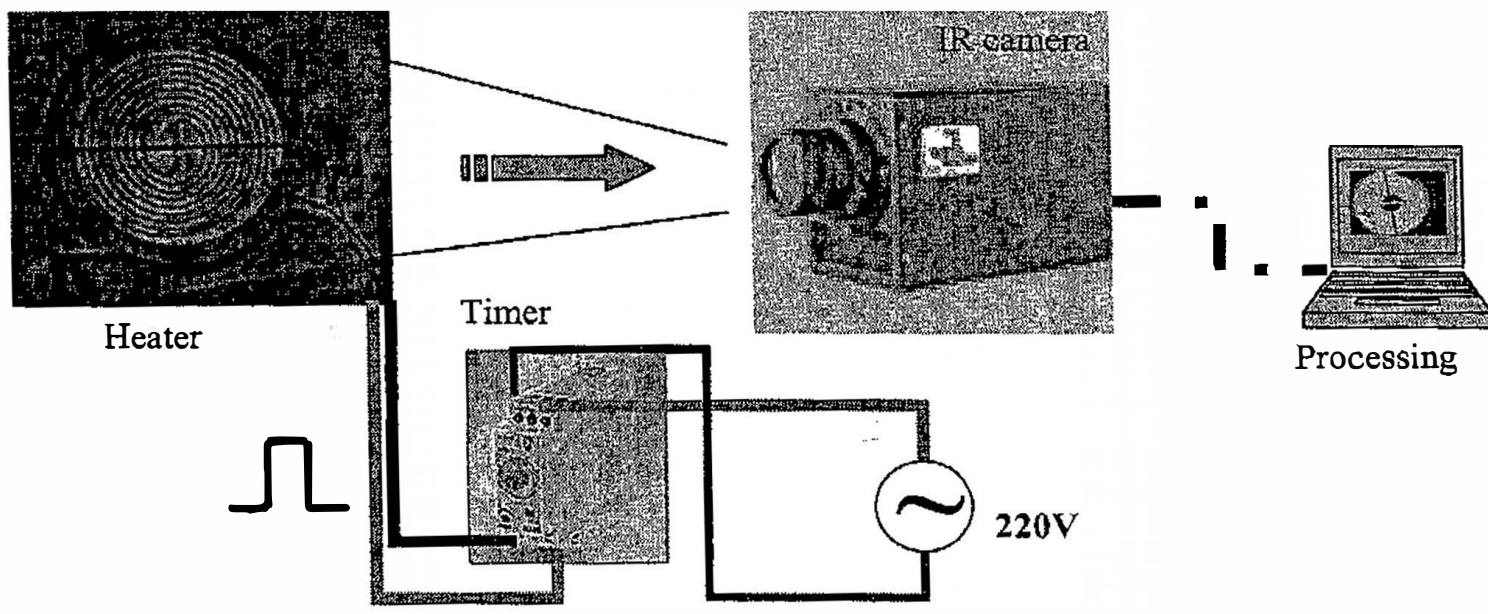

Figure 1. Setup proposed for the on-line quality inspection of radiant heaters.

The heating profile of any pixel reveals a linear dependency with time as represented on Figure $2 \mathrm{~b}$ for pixels selected on Figure 2a. Different pixels exhibit different slopes as a consequence of having dissimilar thermal properties. This fact can be used to discriminate defects in the heater. 

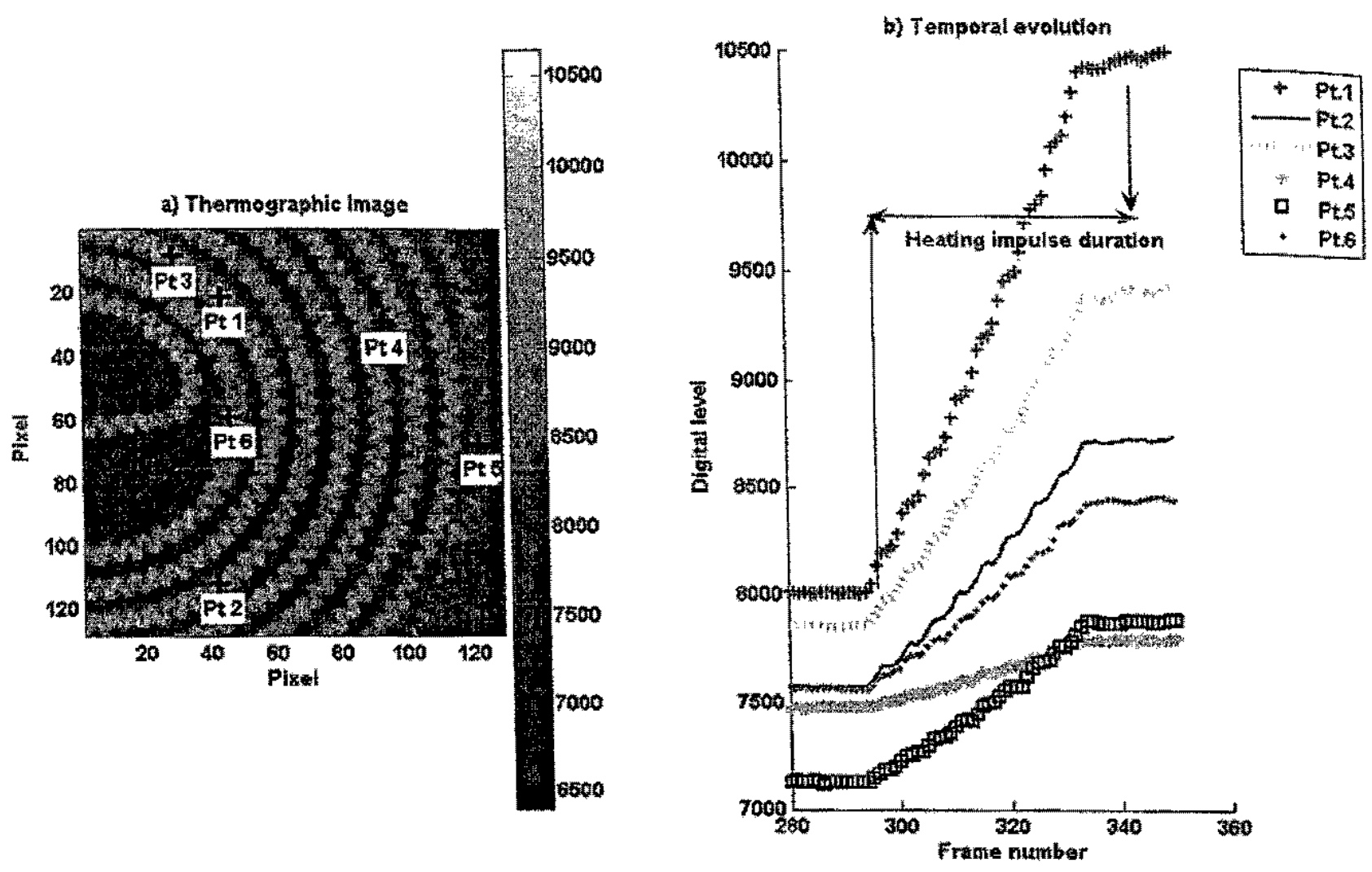

Figure 2. a) Location of pixels under inspection. Pt 1, Pt 5 and Pt 6 are located on defects along the wire element. Pt 2 and Pt 3 belong to defect-free points in the heating element while 4 is on the substrate. b) Digital levels of infrared radiation intensity along the sequence recorded showing a linear temporal evolution.

Once the themographic sequence bas been captured, a simple algorithm has been developed to help in the detection and classification of defects. Its simplicity will lead to a fast inspection, what it is highly interesting for an online production quality control.

\subsection{Processing}

The heating can be approximated by a straight line as it was demonstrated on Figure $2 \mathrm{~b}$. The detection of defects can be carried out just considering the value of the slope on heating tlanks. The thermographic images of interest are those just regarding the heating flank. They can be obtained providing certain synchronization between the electrical pulse source and the camera.

In order to make a correct linear fitting, the exact time of the beginning and end of the impulse must be known. However, this is not an easy task. But the use of specialized transforms applied in image processing and created for the detection of lines in noisy environments can help. To calculate the actual slope of the heating flank, an image processing transform has been studied avoiding the knowledge of the exact frame numbers corresponding to the beginning and end of the impulse: the Radon transform. The Radon Transform (RT) is able to transform two dimensional images with lines into a domain of possible line parameters, where each line in the image will give a peak positioned at the corresponding line parameters. The main characteristic of the Radon transform is its ability to extract lines (curves in general) from very noise images [4]. It is a standard tool in image analysis, which can be implemented on a Matlab ${ }^{\mathrm{TM}}$ code and avoid the need to know the exact tirnes of the start and end of the heating. Figure 3 shows some samples of transformations using Radon Transform. 

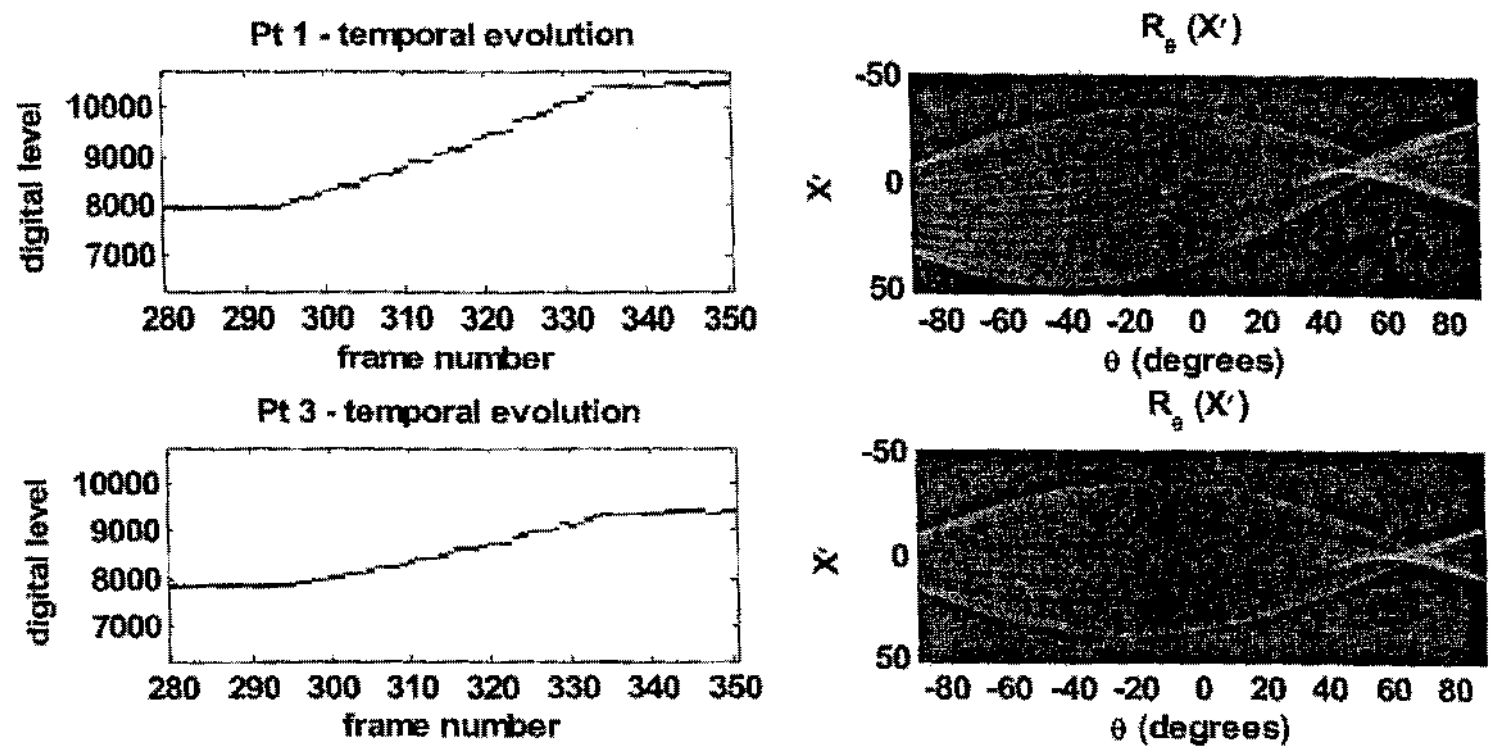

Pt 5 - temporal evolution
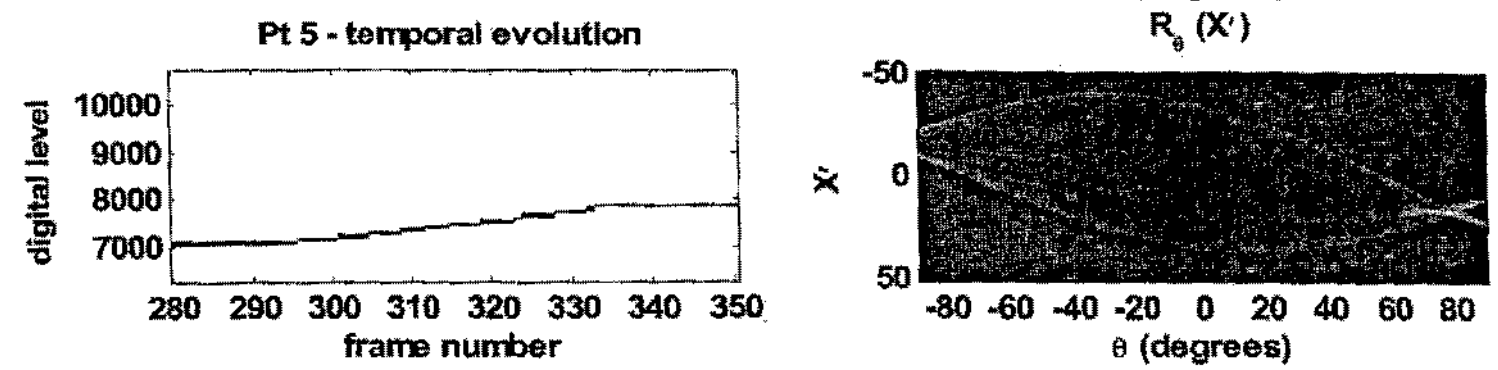

Figure 3. Example of the use of the Radon Transform. In the figures on the second column, the strongest peaks in $R$ give the angle (actually it is the complementary angle) and location of the longest straight segment of the graphs on the frrst column. Graphs in the first column represent different heating slopes of each pixel. The second column lets us see how the angle or slope of the heating profile evolves as the Racion transform is applied.

An automatic inspection process is provided allowing a fast process and independent of any buman intervention. The infrared camera captures a sequence of thermographic images during the quasi instantaneous heating controlled by the electrical timer. A computer processes the sequence using a Radon Transform based code and performs the detection of the defects, highlighting in a single image the points where a dissimilar temporal heating slope exists. An example is tshown on Figure 4. 


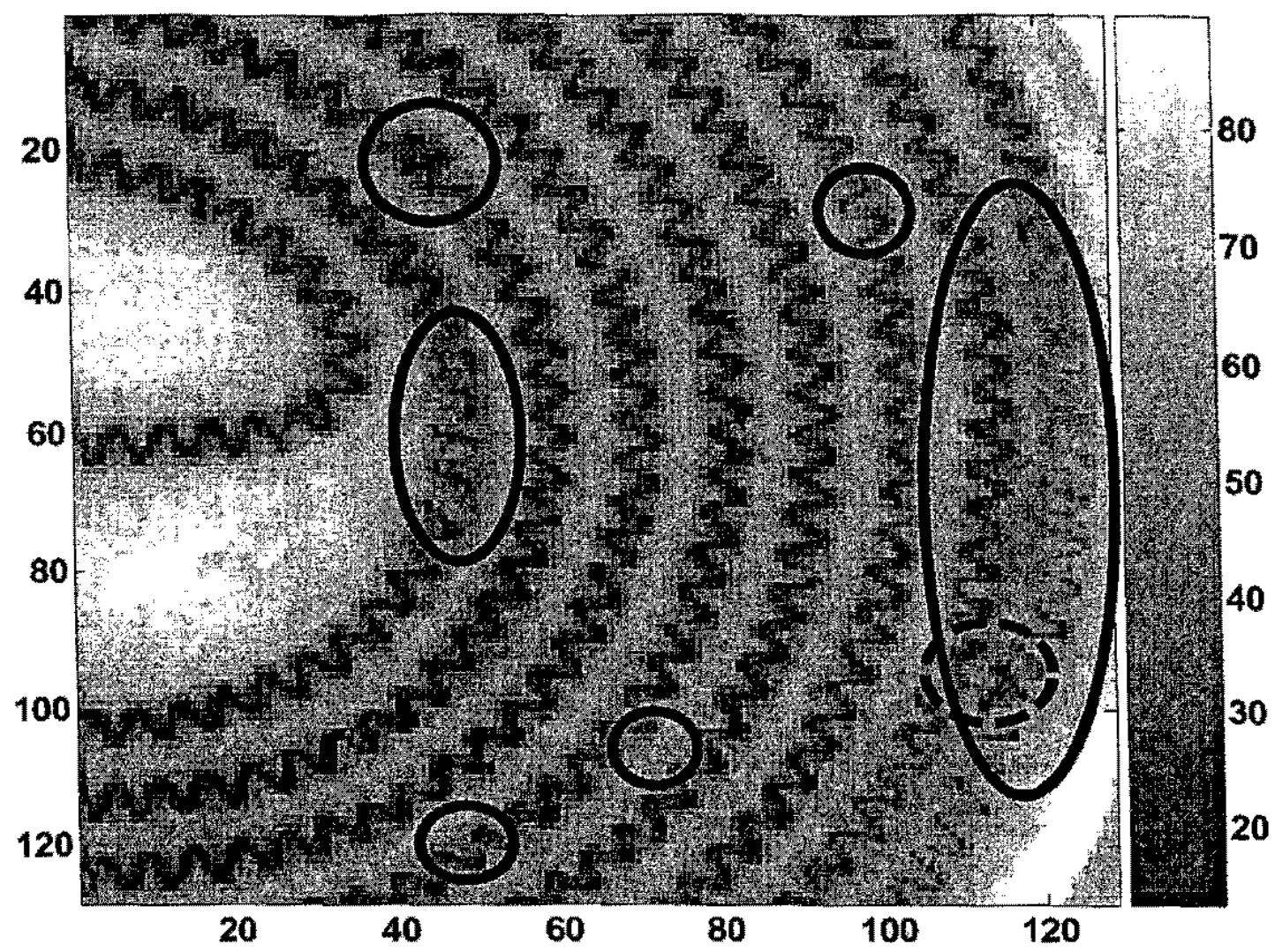

Figure 4. Image containing the complementary angle obtained by means of the Radon transform. Several areas are circled for their analysis. These areas exhibit big slope differences with the areas on their neighbourhood.

\section{RESULTS DISCUSSION}

An operator can quickly locate the potential defects, just with the observation of the single image provided by the algorithm, like the one depicted in Figure 4. Instinctively, some defects can be classified and explained using the appropriate decision threshold. However, for others, their classification is left to operator's experience [2].

Following the Joule's law (the amount of heat per second that develops in a wire carrying a current is proportional to the electrical resistance of the wire and the square of the current), the points where an excess of material exists are depicted in a brighter colour. Having bigger resistance, the temporal heating slope is higher reaching bigger temperatures in less time. Typical defects of this origin are links and doubled or overlapped twists.

Darker points along the wire pattern are related to areas with a lower slope. Using the same reasoning, a lower slope is associated to a lack of material. Therefore, cuts in the heating cable and straight segments are representative of this defect. Also brackets (small metal pieces which anchor the heating cable to the high thermal capacity substrate) are represented by dark areas. However, they don't indicate a lack of material but the attachment of other material in parallel with the heating wire resistance and, therefore, reducing the global resistance in that area. Although they are not strictly defects, brackets should be well placed for the correct fixing of the wire to the substrate and this fact should be also inspected.

Defective fixings of the wire to the substrate are shown as blurring areas. Depending on where the blurring is observed (over the beating element area or between spires), the defective fixing can be classified as a shortage fixing (the wire has an area without substrate underneath) or an excess fixing (part of the substrate is partially burying the heating element). 
Figure 5 shows a collage of graphs where photos on vision-eye range are associated with parts of the processed image obtained after the inspection process described in this paper.

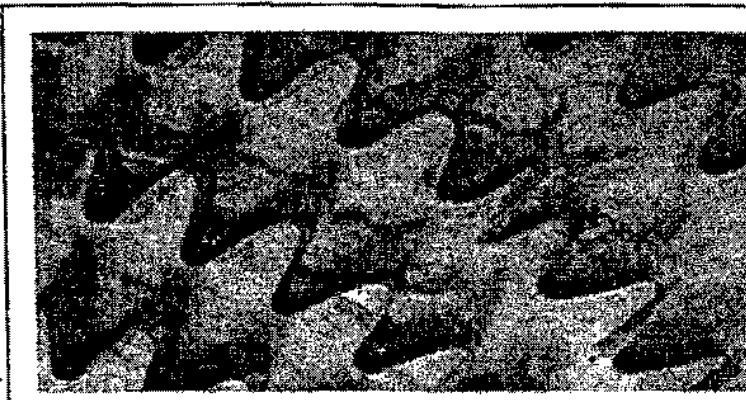

a)

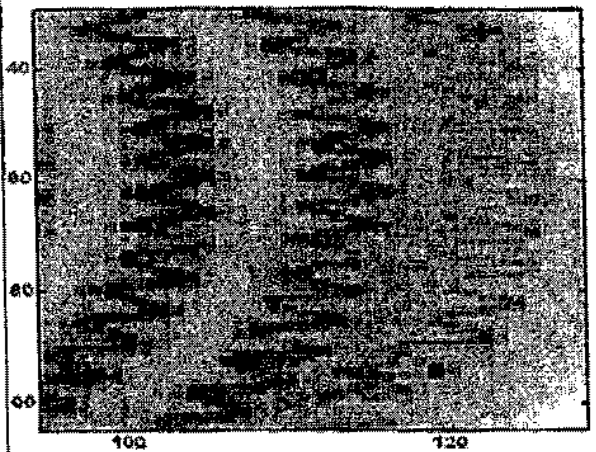

b)

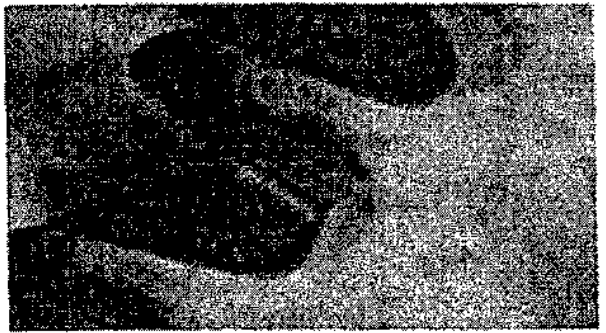

c)

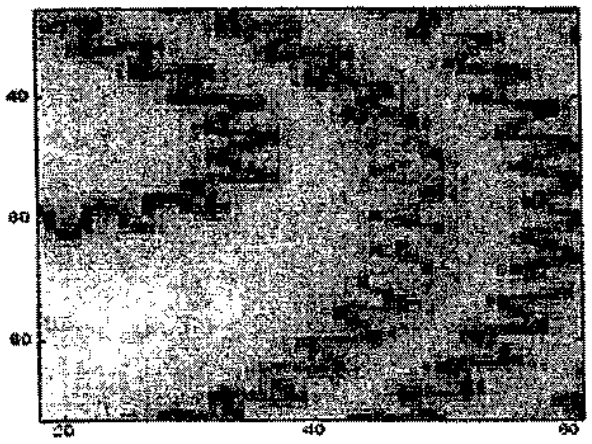

d)
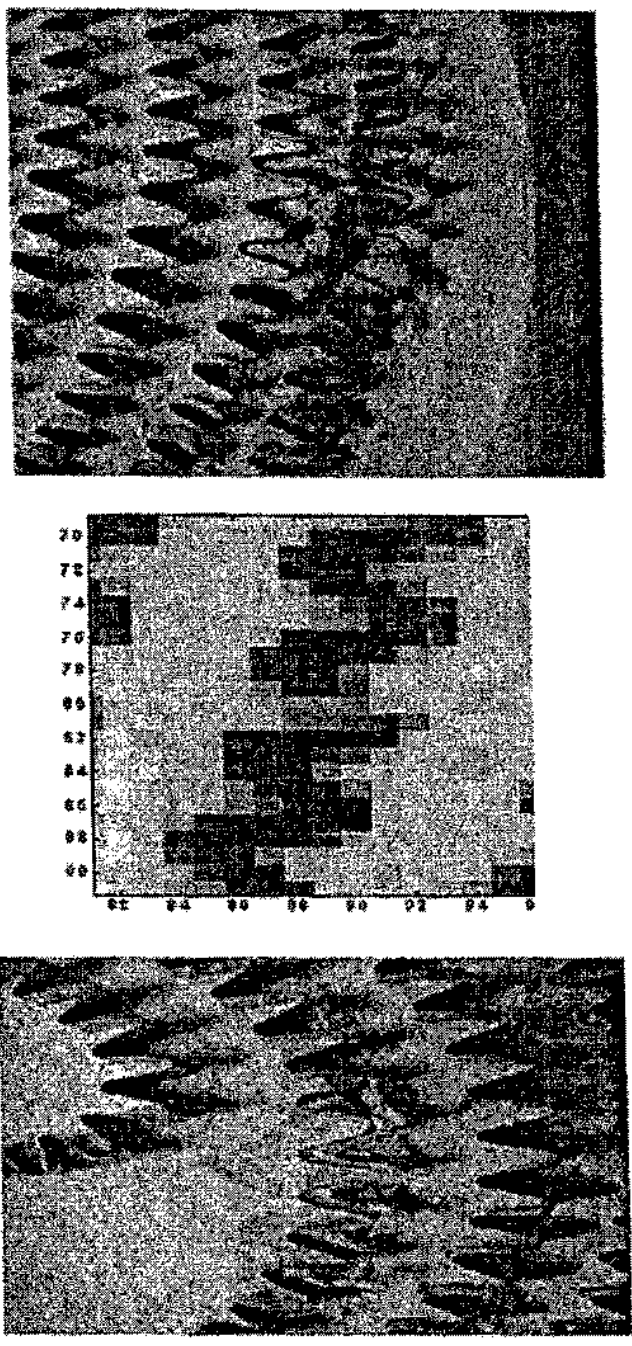

Figure 5 . Real and artificial defects on radiant heaters: a) Excess of material as a consequence of a link. b) and d) parts of the wire that are not in contact with the substrate c) bracket used to attach the heating element to the substrate. 


\section{CONCLUSIONS}

A quality control process on an industry dedicated to radiant heater manufacturing was presented. The effectiveness of Infrared Themography as a NDT\&E technique for this niche of application has been demonstrated. After short pulse excitations in the order of $0.1 \mathrm{sec}$, the recording of thermal innages during the heating and cooling tendencies by a fast infiared camera provides relevant information for the defects that can appear on the production line. A simple defect detection processing which lies in the analysis of the heating-up flank, has been reduced to just a few seconds. A simple processing based on the Radon Transform has been developed to help in the detection and classification of defects. In particular, the range of real and artificial defects in a commercial radiant heater includes: lack of supporting brackets; defects ariginated by a deficiency in the heating material; defects from an excess of heating material; and parts of the heating elements which are in wrong contact (non-contact or semi-buried) with the substrate. All of them have been successfully detected by the non-contact and safe quality control process here presented.

\section{ACKNOWLEDGMENTS}

The Spanish Science and Technology Minister under project SiRAS TEC2004-05936-C02-02 have supported this work. The authors also thank Josı Duoandikoetxea for his practical support in the development of this paper.

\section{REFERENCES}

1. X. Maldague, Theory and Practice of Infrared Thermography for Nondestructive Evaluation, New York, John Wiley pub., 2001, 684p.

2. D.A. González, F.J. Madruga, M.A. Quintela and J.M. López-Higuera, Defect assessment on radiant heaters using infrared thermography, NDT \& E International, Volume 38, Issue 6, September 2005, Pages $428-432$

3. D. A. González , F. J. Madruga, M. Á. Quintela and J. M. López-Higuera, Quality Control of Radiant Heaters, Proceedings of the International Congress on Optics and Optoelectronics, Warsaw, Poland, 2005

4. P. Toft, "The Radon Transform - Theory and Implementation", Ph.D. thesis - Department of Mathematical Modelling, Technical University of Denmark, June 1996. 326 pages. 